\title{
Taxi Drivers' Gestures in Jordan: A Sociolinguistic Approach
}

\section{Dr. Wafa Abu Hatab}

Zarqa University; whatab@zu.edu.jo

\section{Doi:10.5901/mjss.2016.v7n3s1p312}

\section{Abstract}

Taxi drivers in Jordan employ many strategies to communicate while driving using both verbal and nonverbal systems of communication. Nonverbal communication may involve the use of blinkers, headlights, eye gaze, head and hand gestures. The present study examined an aspect of taxi drivers' interpersonal communicative behaviour while driving, namely, use of gestures providing a sociolinguistic analysis of these gestures and taking into consideration the sociolinguistic factors and dimensions that determine their choice. The study was conducted on a random sample consisting of 100 male taxi drivers in Amman, the capital city of Jordan. During the first phase, informants were asked to fill in a questionnaire on the gestures they use while driving or during their breaks. The second phase involved interviews with ten informants. Finally, three informants were videotaped during their breaks. The drivers' responses were then classified into groups according to age, education and experience in taxi driving. Interviews were then transcribed and analyzed in light of the questions of the study and the gestures used by the videotaped drivers were analyzed. The study revealed that age and education of taxi drivers affected the type and frequency of gestures used. Young drivers tended to gesture more while old drivers considered gesturing as an immature linguistic behavior. Old drivers with university education gestured less while drivers with limited school education gestured more. Further research is recommended on identifying the role gender plays in the use of gestures as well as other verbal and nonverbal communicative features of this slice of society

Keywords: gestures, gender, sociolinguistics, status, nonverbal.

\section{Introduction}

The process of human communication is quite complex. When talking, we try to utilize speech to convey information effectively. When we have a conversation with an individual physically present, speech is not the only means employed since the entire body is brought into play. In particular, the head, face, hands and fingers get involved in various types of gestures. Therefore, body language plays an important role in our everyday communication. Albert Mehrabian (1971) found out that the total impact of a message is about 7 per cent verbal, 38 percent vocal and 55 percent non-verbal. This nonverbal communication can come in several forms: body position, eye contact, facial expressions, physical appearance, touch and space. Gesture is one aspect of body language that does not only involve the use of hands but also could involve eye gaze, head, shoulders, legs or even feet but hand gestures are the most widely used. Our bodies are utilized effectively to communicate in a way that facilitates delivering the message and comprehension. When speakers pay attention to the body language of those around, their ability to assess their message will improve considerably. The listener's body language will help you monitor your delivery of an idea or message.

Despite the extensive use of body language in communication, body language as a discipline can be considered as relatively a recent science compared to the other disciplines. Early attempts date back to the1960s that witnessed the emergence of the non verbalist scientists who started to watch non verbal communication in actual social settings. Only in 1970s the science was publicized with the publication of Julius Fast's book in 1970 entitled Body Language.

Every nation has its own body language comprising of a number of gestures people use to convey specific messages. Sometimes young people have their own special gestures that only they and their friends understand. Common gestures are gestures that most people would understand. They stay the same for many years. Gestures can show happiness, encouragement, anger, sadness, boredom, hope, and many other emotions. Certain gestures could vary from one culture to another or even from one occupation to another among the same culture. The present study tackles the use of gestures among taxi drivers in Jordan. The concept of gesture will be identified in the following sections. 


\subsection{Gesture Definition}

Most definitions of gesture are based on Adam Kendon's works who is recognized worldwide for his contributions to this field and for developing a general framework for studding gesture. Kendon (2004:7) defines gestures as the 'visible acts' we employ as a part of how we say things to one another'. This visible act is characterized according to Kendon (2004) by "excursion" thus involving a departure from a baseline and then a return to it. Gesture is an important part of human conversational interaction (McNeill,1992). Kurtenbach and Hulteen (1990) establish a distinction between what constitutes gesture and what is not postulating that "A gesture is a motion of the body that contains information. Waving goodbye is a gesture. Pressing a key on a keyboard is not a gesture because the motion of a finger on its way to hitting a key is neither observed nor significant".

The relation between gesture and speech was tackled from two different perspectives. The first considered gesture as an aid to the speaker that help in clarifying the message when words cannot. This view is adopted by Norbert Freedman (1977), Sotara Kita (1991), Bernard Rimé (2000) and Robert Krauss (1996) and (2000).

The second viewed gestures as basic components of the final product. They are considered as 'partners' to speech in producing the utterance where gesture and speech function together as a coexpressive whole, providing one's interlocutor access to semantic content of the speech act. This view is clear in the works of Kendon (2004) ,McNeill (1992), Calbris (1990), Clark(1996), Gullberg (1998) and de Ruiter (2000) and (2004) .

Gesture is thus a potential resource for interactants as they negotiate social worlds. As Kendon (2000: 51) puts it, "speakers often employ gesture in such a way as to make something that is being said more precise or complete."

"speech and gesture are produced together, and that they must therefore be regarded as two aspects of a single process ... Speakers combine, as if in a single plan of action, both spoken and gestural expression'.' (Kendon 1997: 110-11).

McNeill (1992) and McNeill and Duncan (2000) argue that speech and gesture originate in a single conceptual source. Kendon has concluded that: The gestures that people produce when they talk do play a part in communication and they do provide information to co-participants about the semantic content of the utterances, although there clearly is variation about when and how they do so (Kendon, 1994, p. 192).

For the present study, the researcher defines gesture as

Visible acts that involve using hands, eyes, head, fingers or mouth instead of speech to communicate.

\subsection{Gestural Forms and Classification}

Gesture consists of three stages: preparation, stroke, and recovery. Preparation is the hand's movement from resting position towards a specific point from which the main gesture or stroke can be produced. Stroke is the shape of the gesture as it is completely formed. The movement after the stroke in which the hand moves away and relaxes or returns to a resting position before preparing for the next gesture is known as the recovery. (Kendon 1980, 2000).

Gestures have been categorized into ones that can be recovered from context and those that could not. Gestures that could be recalled were referred to as 'symbolic gestures', Calbris (1990) and Efron(1972), 'emblems' (Ekman and Friesen, 1969) or 'quotable gestures' (Kendon, 1990, 1992). The classification of gestures provided by Kendon (1986) and McNeill (1992) establishes a distinction between what is referred to as 'gesticulation' or the free- gesturing and sign language.

Rime and Schiaratura (1991) classify gestures into symbolic ,deictic, iconic ,and pantomimic gestures. Other types were proposed by McNeill (1992) who used the terms beat gestures and cohesives. Beat or baton gestures involve the up and down hand movements accompanied by speech rhythm. Cohesives, on the other hand, could be iconic, pantomimic or deictic gestures that are used to link related gestures. Iconic and metaphoric gestures are proposed by McNeill (1985; 1992).

\section{Scope of the Study}

Gesture was studied in many languages: Libyan and Moroccan, Barakat (1973) and Brewer (1951), Catalan, Payrató (1993), French, Calbris (1990), Kenyan, Creider (1977), North American and Colombian, Johnson (1975) Iranian, Sparhawk (1981), Russian, Monahan (1983), Brazilian Rector (1986) and Spanish Green (1968).

Gestures studies in the Jordanian context are very rare. The researcher could not locate any study that tackles 
gesture from a linguistic perspective. The present study investigates the use of gesture by a slice of the Jordanian society, namely, taxi drivers.

Taxi drivers in Jordan employ many strategies to communicate their ideas while driving. They might use blinkers, headlights, eye gaze, head and hand gestures. The present study tackles an aspect of taxi drivers' interpersonal communication skill on the road that involves use of gestures to communicate. It aims to provide a sociolinguistic analysis of these gestures taking into consideration the sociolinguistic factors and dimensions that determine the choice of gestures. Studies tackling taxi drivers' gestures in Jordan have never been conducted before. It is expected that the present study can shed some light on the behaviour of a group of people that constitutes a considerable slice of the Jordanian society. Members of this slice are usually referred to as 'the yellow fleet' indicating the vast number of taxi drivers in Jordan. It is hoped that findings of the study can provide more insight into an overlooked area of research, particularly, the pragmatic function of paralinguistic aspects of human interaction.

The questions that the present study tackles can be specifically stated as follows:

1. What gestures are used by taxi drivers? What messages do they convey?

2. How do the age and experience of the taxi drivers affect their use of gestures?

3. What role does education play in determining the gestures used?

\section{Limitations}

Taking into consideration the large number of taxi drivers in Jordan, the study was limited to a random sample of 100 taxi drivers in Amman, the capital. Findings, therefore, cannot be generalized to all taxi drivers in Jordan .Moreover, at the time of data collection, women were not allowed to work as taxi drivers. In 2015, the first female taxi driver was licensed. Consequently, the study was limited to male taxi drivers.

\section{Methodology}

The study provides an analysis of taxi drivers' gestures based on systematic observation and analysis of such gestures in actual use. Without natural communicative data Kendon (1992) argues, we cannot adequately describe the functions these gestures fulfill .The study was conducted on a random sample of taxi drivers in Amman consisting of 100 drivers. Data collection took place over three phases. .During the first phase informants were asked to fill in a questionnaire on the gestures they use while driving or during their breaks. The aim of the questionnaire was to get introduced to the number and variety of gestures used. The second phase involved interviews with ten informants. Finally, three informants were videotaped during their break time. The drivers' responses were then classified into groups according to age, education and experience on the road. Interviews were then transcribed and analyzed in light of the questions of the study. Finally, the gestures used by the videotaped drivers were analyzed.

\section{Analysis and Discussion}

Informants were classified into four groups according to age, in an attempt to investigate the effect of age of the taxi driver on the gestures used. Number of taxi drivers in each age group is displayed in the table below

Table (1). Informants Classified according to Age

\begin{tabular}{|c|c|}
\hline Age Group & Number \\
\hline $20-30$ & 44 \\
\hline $31-40$ & 30 \\
\hline $41-50$ & 14 \\
\hline $51-60$ & 12 \\
\hline Total & 100 \\
\hline
\end{tabular}

These informants were asked to fill in a questionnaire consisting of twenty questions dealing with the drivers' use of gestures to ask for drinks, to give directions, to warn and apologize. Informants' responses were analyzed according to age, education and years of experience. The following table shows the percentages of the use of gesture according to age with regard to the functions outlined in the questionnaire. 
Table (2). Age and Gestures

\begin{tabular}{|c|c|c|c|}
\hline Age & Use of Speech & Gesture & Speech and Gesture \\
\hline $20-30$ & 37 & 53.5 & 9.5 \\
\hline $31-40$ & 30 & 53 & 17 \\
\hline $41-50$ & 47 & 47 & 6 \\
\hline $51-60$ & 50 & 41 & 9 \\
\hline
\end{tabular}

As the figures in the above table show, the highest use of gestures was among drivers from 20-40 years where the gestures constituted almost half of the message. Gestures accompanied by speech were the highest among the drivers whose age was between 31-40.Drivers who were between 51-60 used speech more than gestures. This difference could be attributed to social as well as psychological reasons. The society always expects mature linguistic behavior from the old. The old interviewees said that they did not use gestures when they want to buy coffee, for example, saying that these are the gestures young drivers use. This finding agrees with Labov (1994, p. 107) who postulates that individuals tend to preserve their speech patterns as they move through their lifespan. (Eckert 1997,p. 157) states that "only the middle aged are seen as engaging in mature use, as "doing" language rather than learning it or losing it".

Education was another factor that was investigated. Informants were classified into three groups, drivers with university education, secondary education and basic education (1-10) years at school. Results obtained for drivers with university education are displayed in table 3.

Table (3). Use of Gestures by Drivers with University Education

\begin{tabular}{|c|c|c|c|}
\hline Age & Speech & Gesture & Speech and Gesture \\
\hline $20-30$ & 41.5 & 52.5 & 6 \\
\hline $31-40$ & 41.5 & 46 & 13 \\
\hline $41-50$ & 74 & 23 & 3 \\
\hline $51-60$ & 28 & 46 & 26 \\
\hline
\end{tabular}

The highest percentage for the use of gestures was among university graduates in the age group 20-30 while the lowest was for drivers between 41 and 50 . Gesture and speech were more frequently used among drivers who were between 51-60. Drivers with secondary education showed more tendencies to rely on gestures for communication specially the ones in the age groups 20-30 and 31-40. This might suggest that drivers with higher education tend to gesture less, want to indicate their higher social status by avoiding a linguistic behavior that could stereotype them or simply were unfamiliar with these gestures. The following table displays figures obtained for use of gesture among drivers with secondary education.

Table (4). Use of Gestures by Drivers with Secondary School Education

\begin{tabular}{|c|c|c|c|}
\hline Age & Speech & Gesture & Speech and Gesture \\
\hline $20-30$ & 41.5 & 49.5 & 9 \\
\hline $31-40$ & 25 & 53 & 22 \\
\hline $41-50$ & 29.5 & 68 & 2.5 \\
\hline $51-60$ & 67 & 33 & 0 \\
\hline
\end{tabular}

Drivers with basic education, i.e. drivers who did not finish school and were enrolled for 5-10 years at school, used more gestures than the other two groups. This could suggest that the lower the driver's education level is, the higher the use of gestures will be. Education enriches one's linguistic repertoire, a thing that might reduce reliance on gestures since education opens new social horizons and widens linguistic repertoire. We usually use gestures to aid our communication when words do not help us convey our thoughts. Table (5) shows figures obtained for this group.

Age could also be taken into consideration when discussing use of gestures among basic school drivers. Old drivers, as in other groups, gestured less. The percentage for their use of gesture was $45 \%$, while speech constituted less than $30 \%$ of younger drivers. Gestures were more frequent among young and middle aged drivers of this group with basic education. Such a tendency can be attributed to the desire to belong to the young group and to cope up with the linguistic behavior of the majority. It can be viewed as a strategy of speech accommodation in which young and middle 
aged drivers use gestures as a nonverbal behavior that help them fit in the group of the other young taxi drivers. For old drivers, such a desire is replaced by a desire to fit in with the 'wise' group from the society's perspective. The Jordanian society has its own stereotypes of linguistic behavior of old and young. As a result, old drivers might prefer to abide by social norms rather than rebelling against them.

Table (5). Use of Gestures/Basic Education

\begin{tabular}{|c|c|c|c|}
\hline Age & Speech & Gesture & Speech and Gesture \\
\hline $20-30$ & 27.5 & 58.5 & 14 \\
\hline $31-40$ & 25 & 60 & 15 \\
\hline $41-50$ & 37.5 & 49 & 13.5 \\
\hline $51-60$ & 55 & 45 & 0 \\
\hline
\end{tabular}

By experience, the researcher meant the number of years the drivers have been working as taxi drivers. Experience of taxi drivers in the analyzed sample ranged between one to twenty years. For the first age group (20-30), experiences ranged between 1-10 years. The study revealed that taxi drivers in this group gestured less as they gained more experience. If gestures used to communicate with waiters at coffee shops are taken into consideration, use of less gestures among more experienced drivers might be due to their familiarity with these waiters who know what a familiar driver wants to drink even without saying a word .Waiters are familiar with their regular customers. Notice how the number drops from $49 \%$ for drivers with 1-5 years experience to $37.5 \%$ as shown in table 6 .

Table (6). Experience and Gesture (20-30)

\begin{tabular}{|c|c|c|c|}
\hline Experience & Speech & Gesture & Speech and Gesture \\
\hline $1-5$ & 39 & 49 & 12 \\
\hline $6-10$ & 41.5 & 37.5 & 21 \\
\hline $11-15$ & - & - & - \\
\hline $16-20$ & - & - & - \\
\hline
\end{tabular}

The same argument could apply to the second age group which showed a slight drop in the use of gestures with more experience gained by the taxi drivers whose experience ranged between one and ten years.

Table (7). Experience and Gesture (31-40)

\begin{tabular}{|c|c|c|c|}
\hline Experience & Speech & Gesture & Speech and Gesture \\
\hline $1-5$ & 25 & 61 & 14 \\
\hline $6-10$ & 26 & 59 & 15 \\
\hline $11-15$ & 0 & 0 & - \\
\hline $16-20$ & - & - & - \\
\hline
\end{tabular}

Compared to this group, the third group, 41- 50 displayed a tremendous continuous drop concerning use of gestures from 67.5 to 23 as shown in table 8.

Table (8). Experience and Gesture (41-50)

\begin{tabular}{|c|c|c|c|}
\hline Experience & Speech & Gesture & Speech and Gesture \\
\hline $1-5$ & 17.5 & 67.5 & 15 \\
\hline $6-10$ & 53 & 39 & 8 \\
\hline $11-15$ & 64.5 & 33.5 & 2 \\
\hline $16-20$ & 73.5 & 23 & 3.5 \\
\hline
\end{tabular}

Long experience led to a reduction in the number of gestures used among the most experienced drivers. Drivers with ten years experience relied mainly on speech and did not use gestures with speech. Gestures were used for $37.5 \%$ that dropped to $17 \%$ among drivers whose experience ranged between 11-15 then rose to $30 \%$ among drivers with 16-20 
years in taxi driving. Figures are displayed in table 9.

Table (9). Experience and Gesture (51-60)

\begin{tabular}{|c|c|c|c|}
\hline Experience & Speech & Gesture & Speech and Gesture \\
\hline $1-5$ & - & - & - \\
\hline $6-10$ & 62.5 & 37.5 & 0 \\
\hline $11-15$ & 58 & 17 & 25 \\
\hline $16-20$ & 63 & 30 & 7 \\
\hline
\end{tabular}

\section{Conclusion}

The present study investigated the use of gestures by taxi drivers in Jordan adopting a sociolinguistic approach. Data were collected from Amman, the capital city covering a random sample of 100 male drivers. Analysis revealed that age and education of taxi drivers affected the type and frequency of gestures used. Young drivers tended to gesture more while old drivers considered gesturing as immature linguistic behavior. Furthermore, education affected the gesturing practice. Old drivers with university education gestured less while drivers with limited school education gestured more.

The study was conducted on a small sample so findings cannot be generalized to all taxi drivers in Jordan. Further research is recommended to explore regional differences in the use of gestures among taxi drivers and other drivers in Jordan. Since female taxi drivers have recently joined the 'yellow fleet', more research is recommended on identifying the role gender plays in the use of gestures as well as other verbal and nonverbal communicative features of this slice of society. Findings of the present study can trigger future research that compares and contrasts the linguistic behavior of taxi drivers in Jordan with other Arab and foreign countries.

\section{References}

Barakat, Robert A.(1973). Arabic Gestures. Journal of Popular Culture 6(4):749-792.

Beattie, G. \& Shovelton. H. (1999). Mapping the range of information contained in the iconic hand gestures that accompany spontaneous speech. Journal of Language and Social Psychology, 18, 438-462

Brewer, W.D. (1951). Patterns of Gesture among the Levantine Arabs. Amercian Anthropologist 53:232-237.

Calbris, G. (1990). Semiotics of French Gesture. Bloomington: Indiana University Press.

Clark, H. H. (1996). Using Language. Cambridge: Cambridge University Press.

Creider, Chet A. 1977 Towards a Description of East African Gestures. Sign Language Studies 14:1-20.

De Ruiter, J. P. (2000). The production of gesture and speech. In D. McNeill (Ed.), Language and Gesture. (pp. 284-311). Cambridge: Cambridge University Press.

De Ruiter, J. P. (2007). Postcards from the mind. Gesture. 7(1), 21-38

Melinger, A. \& Levelt, W. M. (2004). Gesture and the communicative intention of the speaker. Gesture, 4(2), 119-141

Green, Jerald R. (1968). A Gesture Inventory for Teaching Spanish. New York: Clinton Books.

Gullberg, M. (1998). Gesture as a Communication Strategy in Second Language Discourse: A Study of Learners of French and Swedish. Lund: Lund University Press.

Gullberg, M. \& Holmqvist, K. (2006). What speakers do and what listeners look at. Visual attention to gestures in human interaction live and on video. Pragmatics and Cognition, 14(1), 53-82

Eckert, Penelope (1997) Age as a sociolinguistic variable, in: The Handbook of Sociolinguistics, Coulmas, Florian, ed., Oxford, 151-167.

Ekman, Paul, Friesen, Wallace. (1969). The repertoire of non-verbal behavior: Categories, origins, usage and coding. Semiotica 1(1), 49-98.

Freedman, N. (1977). Hands, words and mind: On the structuralization of body movements during discourse and the capacity for verbal representation. In N. Freedman \& S. I. Grand (Eds.), Communicative Structures and Psychic Structures: A Psychoanalytic Approach. (pp. 109-132). New York and London: Plenum Press

Johnson H.G., Paul Ekman, and Wallace Friesen .( 1975 ). Communicative Body Movements: American Emblems. Semiotica 15:335353.

Kendon, Adam. (1980). Gesticulation and speech: Two aspects of the process of utterance.In Key, Mary, R. (Ed.).(1980). The relationships of verbal and nonverbal communication, Mouton, The Hague, pp.207-227.

Kendon, A. (1983). Gesture and speech: How they interact. In J. M. Weimann \& R. J. E.

Kendon, Adam.( 1990). Gesticulation, quotable gestures, and signs. Senri Ethnological Studies 27, 53-77.

Kendon, A. (1992). Some Recent Work from Italy on Quotable Gestures (Emblems). Journal of Linguistic Anthropology 2(1): 92-108.

Kendon, A. (1994). Do gestures communicate? A review. Research on Language and Social Interaction, 27, 175-200.

Kendon, A. (1997). Gesture. Annual Review of Anthropology 26: 109-128.

Kendon, A. (2000). Language and Gesture: Unity or Duality? In D. McNeill (ed.), Language and Gesture (pp. 47-63). Cambridge: 
Cambridge University Press.

Efron David, 1972[1941]. Gesture, race, and culture. Mouton, The Hague.

Kendon, A (2004). Gesture: Visible Action as Utterance. Cambridge: Cambridge University Press.

Kita, S. (2000). How representational gestures help speaking. In D. McNeill (Ed.), Language and Gesture. (pp. 162-185). Cambridge: Cambridge University Press.

Krauss, R., Chen, Y., \& Chawla, P. (1996). Nonverbal behavior and nonverbal communication: Whatdo conversational hand gestures tell us? In M. Zanna (Ed.), Advances in Experimental SocialPsychology: Volume 28. (pp. 389-450). New York: Academic Press.

Krauss, R. M., Chen, Y., \& Gottesman, R. F. (2000). Lexical gestures and lexical access: a process model. In D. McNeill (Ed.), Language and Gesture. (pp. 261-283). Cambridge: Cambridge University Press.

Kurtenbach, G. \& Hulteen, E. (1990). Gestures in Human-Computer Communications. In B.Laurel (Ed.) The Art of Human Computer Interface Design. Addison-Wesley, 309-317.

McNeill, D. (1985). So you think gestures are nonverbal? Psychological Review, 92, 350-371.

Labov, William (1994) Principles of Linguistic Change. Volume 1: Internal Factors, Oxford.

McNeill, D. (1987). Psycholinguistics: A new approach. New York: Harper \& Row.

McNeill, D. (1992). Hand and Mind: What Gestures Reveal about Thought. Chicago: University of Chicago Press.

McNeill, D., and Duncan, S. (2000). Growth Points in Thinking for Speaking. In D. McNeill (ed.), Language and Gesture (pp. 141-161). Cambridge: Cambridge University Press.

McNeill, D. (ed.) (2000). Language and Gesture. Cambridge: Cambridge University Press

Mehrabian, A. (1971) Silent Message .Wadsworth. Belmont, California.

Monahan, Barbara . (1983) . A Dictionary of Russian Gesture. Ann Arbor, Michigan: Hermitage.

Payrató, Lluís (1993). A Pragmatic View on Autonomous Gestures: A First Repertoire of Catalan Emblems. Journal of Pragmatics 20:193-216.

Rector, M. (1986). Emblems in Brazilian Culture. In Iconicity. Essays on the Nature of Culture. P. Bouissac, M. Herzfeld and R. Posner, eds. Pp. 447-462. Tübingen: Stauffenburg.

Rimé, B. \& Schiaratura, L. (1991). Gesture and speech. In R. S. Feldman \& B. Rimè (Eds.), Fundamentals of Nonverbal Behavior. (pp. 239-281). Cambridge: Cambridge University Press.

Sparhawk, Carol M. (1977) Contrastive-identificational Features of Persian Gestures. Semiotica 24(1/2):49-86.

Wiemann, J.E \& R. P. Harrison (Eds.) (1983). Nonverbal interaction. Beverly Hills, CA: Sage.

\section{Appendix}

Most common Gestures Used by Taxi drivers to Ask for drinks while driving
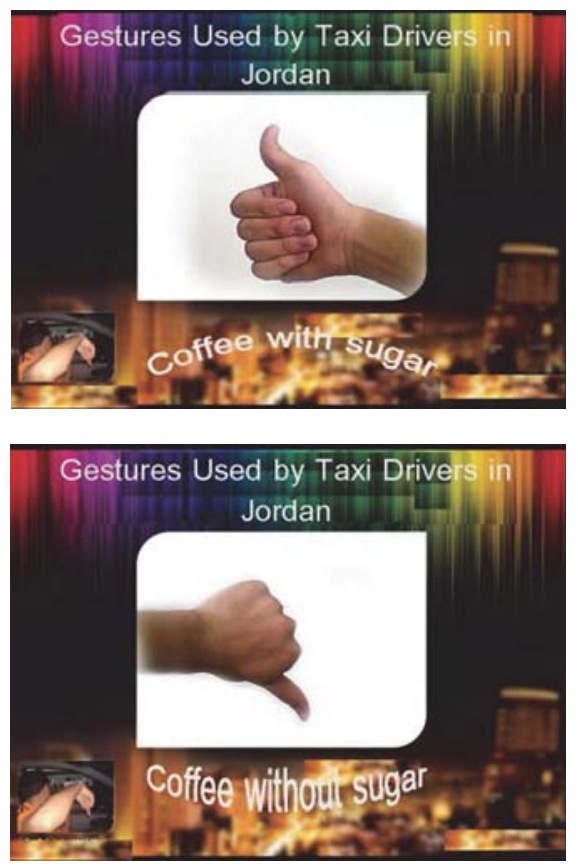

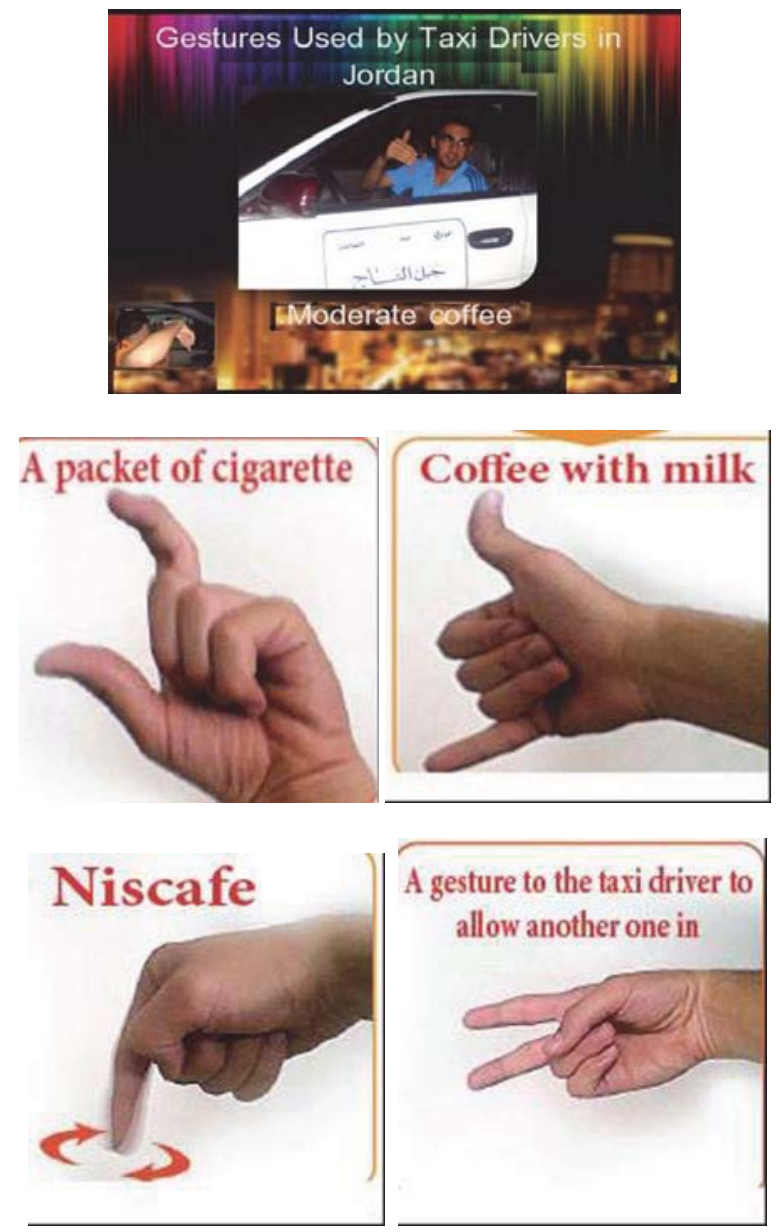